\title{
A COMPARISON BETWEEN INSULIN RESISTANCE SCORES PARAMETERS IN IDENTIFYING PATIENTS WITH METABOLIC SYNDROME
}

\author{
ANGELA COZMA ${ }^{\mathrm{a}, \dagger}$, ADRIANA FODOR ${ }^{\mathrm{b}, \dagger}$, OLGA HILDA ORĂȘAN ${ }^{\mathrm{a}, *}$, \\ RAMONA SUHAROSCHI', CRINA MUREȘAN ${ }^{c}$, ROMANA VULTURAR ${ }^{\mathrm{d}}$, \\ DOREL SAMPELEAN ${ }^{\mathrm{a}}$, VASILE NEGREAN ${ }^{\mathrm{a}}$, DANA POP ${ }^{\mathrm{e}}$, \\ ADELA SITAR-TĂUT ${ }^{a}$
}

\begin{abstract}
As a consequence of the epidemic of obesity, prediction of metabolic syndrome (MetS) is relevant because of its subsequent association with type 2 diabetes and cardiovascular disease. Also, MetS is considered a state of insulin resistance (IR). This study aimed to evaluate the capacity of different indirect IR scores to identify patients with MetS, compared to standard criteria of MetS diagnosis. To the best of our knowledge, the cut-off values of these indexes, as indicators of MetS, in the Romanian population, have not been established. We used a non-parametric extension of the induced ROC regression methodology to determine the cut-off values of HOMA-IR, QUICKI, McAuley indexes, taking into account the MetS components instead of using 90th percentile criteria. Although HOMA is more commonly used in practice (probably due to its easy use), McAuley Score seems to have a better specificity in identifying patients with MetS. This is different between men and women; if in women, McAuley index has the highest sensitivity, in men, HOMA and QUICKI indexes have the highest sensitivity and specificity.
\end{abstract}

Keywords: insulin, glycemia, HOMA, QUICKI, McAuley, metabolic syndrome

a University of Medicine and Pharmacy "Iuliu Hatieganu", 4th Internal Medicine Department Cluj-Napoca, Romania

b University of Medicine and Pharmacy "Iuliu Hatieganu" Clinical Center of Diabetes, Nutrition and Metabolic Disease, Cluj-Napoca, Romania

c University of Agricultural Sciences and Veterinary Medicine of Cluj-Napoca, Faculty of Food Science \&Technology

d University of Medicine and Pharmacy "Iuliu Hatieganu", Department of Cell Biology, ClujNapoca, Romania

e University of Medicine and Pharmacy "Iuliu Hatieganu", Department of Cardiology, ClujNapoca, Romania fauthors with equal contribution

*Corresponding author: E-mail: olgaorasan@yahoo.com 
A. COZMA, A. FODOR, O. H. ORĂȘAN, R. SUHAROSCHI, C. MUREȘAN, R. VULTURAR,

D. SAMPELEAN, V. NEGREAN, D. POP, A. SITAR-TĂUT

\section{INTRODUCTION}

Metabolic Syndrome (MetS) is associated with increased cardiovascular risk, being a common cause of atherosclerotic vascular disease. Also, MetS is considered a state of insulin resistance (IR).

Insulin resistance causes increased atherogenesis and atherosclerotic plaque instability by inducing proinflammatory activity in vascular and immune cells [1,2]. Insulin resistance enhances the risk of developing type 2 diabetes mellitus and is an independent risk factor for major cardiovascular events in patients with preexisting arterial disease [3,4]. Apart from influencing clinical disease development and mortality [5], IR is now recognized to play an important role in preclinical (silent) CAD. According to recent studies, IR is associated with asymptomatic myocardial perfusion defects in normotensive adults with preclinical diabetes [6] and is linked to angiographically documented silent CAD in patients with type 2 diabetes[7,8].

The quantification of IR can be achieved by evaluating peripheral insulin sensitivity in vivo with methods such as the hyperinsulinemiceuglycemic clamp technique or the intravenous glucose tolerance test. They are complicated, time-consuming and expensive methods, unsuitable for clinical studies. Simpler indirect methods have been promoted for quantification of IR. Moreover, several components of MetS (dysglycemia, abdominal obesity, dyslipidemia) are pathophysiologically related to IR.

This study aimed to evaluate the capacity of different indirect IR scores to identify patients with MetS, compared to standard criteria of MetS diagnosis [9]. The homeostatic model assessment of IR (HOMA-IR) is a mathematical model that predicts insulin sensitivity, measured by fasting blood glucose and basal insulin. It is strongly correlated with the hyperinsulinemic-euglycemic clamp $(r=0.88)$, the gold standard for assessing insulin resistance (IR) [10]. It is also correlated positively with the components of MetS such as central obesity, lipid abnormalities and hypertension. However, there is great variability in the threshold HOMA-IR levels to define IR. Most studies have determined the cut-off values based on the percentile criteria (80th or 90th) of values in the general population.

Quantitative insulin sensitivity check index (QUICKI) can be determined from a fasting blood sample and correlates with glucose clamp measures of insulin sensitivity $(r=0.78)$ [11]. QUICKI is similar to HOMA, except that QUICKI transforms the data by taking both the logarithm and the reciprocal of the glucose-insulin product, thus slightly skewing the distribution of fasting insulin values. Moreover, the two methods correlate well.

McAuley index is a score based on a weighted combination of insulin and triglycerides, corrected for weight, determined from a fasting blood sample [12].It is used for predicting IR in normoglycemic individuals. 
IR probability score (IR-PS) is based on insulin, C-peptide, creatinine, TG/HDL-C, and BMI. It has been recently used to assess IR in apparently healthy individuals [13].

The use of these indexes in clinical practice is limited because of the absence of reference values for normal and impaired insulin sensitivity. HOMA-IR and QUIKI are suitable for clinical use, while McAuley index is suitable for epidemiological studies.

To the best of our knowledge, the cut-off values of these indexes, as indicators of MetS, in the Romanian population, have not been established. We used a non-parametric extension of the induced ROC regression methodology [14] to determine the cut-off values of HOMA-IR, QUICKI, McAuley indexes, taking into account the MetS components instead of using 90th percentile criteria. The purpose of this study was to evaluate the capacity of different IR scores to identify patients with MetS, compared to standard criteria of MetS diagnosis.

\section{RESULTS AND DISCUSSION}

The general characteristics of the studied group, including 84 subjects of which 21 men and 63 women with a mean age of $56.89 \pm 11.05$ years, are shown in Table 1. Mean values for systolic and diastolic blood pressure, BMI, waist circumference, plasma leptin, total cholesterol, triglycerides, HDL cholesterol, fasting glucose, $\mathrm{HbA} 1 \mathrm{c}$ and indirect insulin resistance indexes (HOMA, QUICKI, McAuley) are included in these data. Statistically significant differences between the patients with and without MetS were found for age, obesity, abdominal circumference, BMI, hypertension, diabetes, systolic blood pressure, diastolic blood pressure, triglycerides, insulin, HOMA, QUICKI, McAuley indexes.

Patients with MS presented higher insulin resistance (estimated through HOMA index 1.59 vs. $1.04, p=0.0001$ and McAuley Score $2.37 \pm 0.43$ vs. $1.89 \pm 0.36, p=0.0001)$ and lower insulin sensitivity $(0.35 \pm 0.026$ vs. $0.37 \pm 0.026$, $\mathrm{p}=0.0001)$.

HOMA was significantly correlated with BMI (correlation coeff $=0.372$, $p<0.001$ ), SBP (correlation coeff $=0.250, p=0.022$ ), abdominal circumference (correlation coeff $=0.464, p<0.001$ ), glycemia (correlation coeff $=0.592$, $p<0.001$ ), the presence of diabetes (correlation coeff $=0.403, p<0.001$ ), serum TG (correlation coeff $=0.432, p<0.001$ ), the presence of MS (correlation coeff $=0.443, p<0.001$ ), and was inversely correlated with the HDL-cholesterol value (correlation coeff $=-0.287, p=0.008$ )

Performing multivariate regression analysis for HOMA we have found that diabetes was the only independent predictors for HOMA (coefficient of determination $=3.393, p<0.001)($ Table 2$)$. 
A. COZMA, A. FODOR, O. H. ORĂȘAN, R. SUHAROSCHI, C. MURESAN, R. VULTURAR,

D. SAMPELEAN, V. NEGREAN, D. POP, A. SITAR-TĂUT

Table 1. - Characteristics of the studied group

\begin{tabular}{|c|c|c|c|c|c|}
\hline & & Total & $\begin{array}{l}\text { Without } \\
\text { MetS }\end{array}$ & MetS & p \\
\hline Number & No (\%) & $84(100)$ & $29(34.5)$ & $55(65.5)$ & 0.0064 \\
\hline Age & Mean $\pm S D$ & $56.89 \pm 11.05$ & $53.10 \pm 10.69$ & $58.89 \pm 10.80$ & 0.021 \\
\hline Sex & No $(\%)$ & & & & \multirow{3}{*}{0.0118} \\
\hline $\mathbf{F}$ & & $63(75)$ & $27(93.10)$ & $36(65.45)$ & \\
\hline $\mathbf{M}$ & & $21(25)$ & $2(6.9)$ & $19(34.54)$ & \\
\hline Smoking & No (\%) & & & & \\
\hline Yes & & $21(25)$ & $11(37.9)$ & $10(18.18)$ & 0.08 \\
\hline No & & $63(75)$ & $18(62.1)$ & $45(81.81)$ & \\
\hline Obesity & No (\%) & & & & \\
\hline Yes & & 32 (38.1) & $6(20.7)$ & $26(47.3)$ & 0.0316 \\
\hline No & & $52(61.9)$ & $23(79.3)$ & $29(52.7)$ & \\
\hline AC & Mean \pm SD & $96.57 \pm 12.42$ & $89.55 \pm 11.60$ & $100.27 \pm 11.27$ & 0.0001 \\
\hline BMI & Mean \pm SD & $28.81 \pm 4.22$ & $26.89 \pm 4.42$ & $29.82 \pm 3.78$ & 0.0021 \\
\hline HTN & No $(\%)$ & & & & \\
\hline Yes & & $54(64.3)$ & $13(44.8)$ & $41(74.6)$ & 0.0138 \\
\hline No & & $30(35.7)$ & $16(55.2)$ & $14(25.4)$ & \\
\hline Diabetes & No (\%) & & & & \\
\hline Yes & & $13(15.5)$ & $0(0)$ & $13(23.6)$ & 0.0114 \\
\hline No & & $71(84.5)$ & $29(100)$ & $42(76.4)$ & \\
\hline CVD & No (\%) & & & & \\
\hline Yes & & $20(23.8)$ & $7(24.1)$ & $13(23.6)$ & NS \\
\hline No & & $64(76.2)$ & $22(75.8)$ & $42(76.4)$ & \\
\hline Glycemia & Mean $\pm S D$ & $97.35 \pm 16.76$ & $86.75 \pm 8.30$ & $102.94 \pm 17.43$ & $<0.0001$ \\
\hline Total cholesterol & Mean \pm SD & $218.09 \pm 43.58$ & $221.41 \pm 35.86$ & $216.34 \pm 47.36$ & Ns \\
\hline LDL cholesterol & Mean $\pm S D$ & $139.82 \pm 37.05$ & $148.13 \pm 30.30$ & $135.43 \pm 39.71$ & Ns \\
\hline HDL cholesterol & Mean $\pm S D$ & $47.15 \pm 8.5$ & $51.68 \pm 6.93$ & $44.76 \pm 8.32$ & 0.0002 \\
\hline TG & Mean \pm SD & $155.52 \pm 78.01$ & $108.17 \pm 38.30$ & $180.49 \pm 82.19$ & $<0.0001$ \\
\hline SBP & Mean \pm SD & $136.42 \pm 19.67$ & $127.58 \pm 16.4$ & $141.09 \pm 19.78$ & 0.0023 \\
\hline DBP & Mean $\pm S D$ & $84.82 \pm 10.33$ & $80.34 \pm 8.85$ & $87.18 \pm 10.35$ & 0.0034 \\
\hline Insulin* & Mean $\pm S D$ (median) & $7.58 \pm 5.18(5.7)$ & $6.22 \pm 4.05(4.8)$ & $\begin{array}{l}8.29 \pm 5.59 \\
(6.5)\end{array}$ & 0.0014 \\
\hline HOMA index * & Mean $\pm S D$ (median) & $\begin{array}{l}1.86 \pm 1.47 \\
(1.36)\end{array}$ & $\begin{array}{l}1.34 \pm 0.91 \\
(1.04)\end{array}$ & $\begin{array}{l}2.14 \pm 1.63 \\
(1.59)\end{array}$ & 0.0001 \\
\hline HOMA BETA* & Mean $\pm S D$ (median) & $\begin{array}{l}88.61 \pm 56.36 \\
(73.24)\end{array}$ & $\begin{array}{l}98.59 \pm 60.73 \\
(78.10)\end{array}$ & $\begin{array}{l}83.52 \pm 53.86 \\
(69.18)\end{array}$ & NS \\
\hline QUICKI index & Mean $\pm S D$ & $0.35 \pm 0.02$ & $0.37 \pm 0.026$ & $0.35 \pm 0.026$ & 0.0001 \\
\hline Glycemia/insulin & Mean \pm SD & $15.88 \pm 6.02$ & $16.87 \pm 5.43$ & $15.36 \pm 6.30$ & NS \\
\hline McAuley index & Mean \pm SD & $2.06 \pm 0.45$ & $2.37 \pm 0.43$ & $1.89 \pm 0.36$ & 0.0001 \\
\hline
\end{tabular}

${ }^{*}$ Not meeting the normality condition; for normal distribution data Student test was used; for not-normally distributed data Mann-Whitney test was used, for categorical data - $X 2$ test was used; AC - abdominal circumference, BMI- body mass index, HTN - hypertension, CVDcardiovascular disease, TG - triglycerides, SBP - systolic blood pressure, DBP - diastolic blood pressure 
A COMPARISON BETWEEN INSULIN RESISTANCE

SCORES PARAMETERS IN IDENTIFYING PATIENTS WITH METABOLIC SYNDROME

Table 2. - Multivariate regression analysis for HOMA - model summary and coefficients

\begin{tabular}{|c|c|c|c|c|c|}
\hline \multicolumn{5}{|c|}{ Model Summary } & \\
\hline Model & $\mathrm{R}$ & R Square & $\begin{array}{l}\text { Adjusted R } \\
\text { Square }\end{array}$ & $\begin{array}{l}\text { Std. Error of } \\
\text { the Estimate }\end{array}$ & a. Predictors: (Constant), SBP \\
\hline 1 & $.072^{\mathrm{a}}$ & .005 & -.007 & 1.47549 & b. Predictors: (Constant), SBP, AC \\
\hline 2 & $.227^{\mathrm{b}}$ & .052 & .028 & 1.44949 & $\begin{array}{l}\text { C. Predictors: (Constant), SBP, AC, } \\
\text { DM_NO }\end{array}$ \\
\hline 3 & $.467^{\mathrm{c}}$ & .218 & .188 & 1.32472 & $\begin{array}{l}\text { d. Predictors: (Constant), SBP, AC, } \\
\text { DM_NO, TG }\end{array}$ \\
\hline 4 & $.503^{\mathrm{d}}$ & .253 & .215 & 1.30242 & $\begin{array}{l}\text { e. Predictors: (Constant), SBP, AC, } \\
\text { DM NO, TG, HDL }\end{array}$ \\
\hline 5 & $.505^{\mathrm{e}}$ & 255 & 207 & 1.30924 & $\begin{array}{l}\text { f. Predictors: (Constant), SBP, AC, } \\
\text { DM_NO, TG, HDL, MS_NO }\end{array}$ \\
\hline 6 & $.506^{f}$ & 256 & 198 & 1.31701 & g. Dependent Variable: $\mathrm{HOMA}$ index \\
\hline
\end{tabular}

\begin{tabular}{|c|c|c|c|c|c|c|}
\hline & & & Coeffici & & & \\
\hline & del & Unstandard & d Coefficients & Standardized Coefficients & $\mathrm{t}$ & Sig. \\
\hline & & & Std. Error & Beta & & \\
\hline 1 & (Constant) & 1.135 & 1.135 & & 1.000 & .320 \\
\hline & SBP & .005 & .008 & .072 & .654 & .515 \\
\hline 2 & (Constant) & -.552 & 1.400 & & -.394 & .694 \\
\hline & SBP & -.002 & .009 & -.029 & -.246 & .806 \\
\hline & AC & .028 & .014 & .238 & 1.992 & .050 \\
\hline 3 & (Constant) & .628 & 1.311 & & .479 & 633 \\
\hline & SBP & -.006 & .008 & -.082 & -.744 & 459 \\
\hline & AC & .019 & .013 & .159 & 1.429 & 157 \\
\hline & DM_NO & 1.708 & .415 & .423 & 4.120 & .000 \\
\hline 4 & (Constant) & .462 & 1.292 & & .358 & .722 \\
\hline & SBP & -.006 & .008 & -.077 & -.711 & .479 \\
\hline & AC & .014 & .013 & .120 & 1.080 & 283 \\
\hline & DM_NO & 1.483 & .424 & .367 & 3.497 & .001 \\
\hline & $\mathrm{TG}^{-}$ & .004 & .002 & .202 & 1.940 & .056 \\
\hline 5 & (Constant) & .938 & 1.716 & & .547 & .586 \\
\hline & SBP & -.005 & .008 & -.073 & -.669 & .506 \\
\hline & AC & .013 & .013 & .111 & .978 & .331 \\
\hline & DM_NO & 1.497 & .427 & .370 & 3.502 & .001 \\
\hline & TG & .004 & .002 & .187 & 1.675 & .098 \\
\hline & $\mathrm{HDL}$ & -.008 & .018 & -.045 & -.424 & .672 \\
\hline 6 & (Constant) & .967 & 1.730 & & .559 & .578 \\
\hline & SBP & -.006 & .008 & -.080 & -.711 & .479 \\
\hline & AC & .012 & .014 & .104 & .897 & .373 \\
\hline & DM_NO & 1.477 & .435 & .366 & 3.393 & .001 \\
\hline & $\mathrm{TG}^{-}$ & .003 & .002 & .178 & 1.531 & .130 \\
\hline & HDL & -.006 & .019 & -.037 & -.326 & .745 \\
\hline & MS NO & .110 & .385 & .036 & .287 & .775 \\
\hline
\end{tabular}

SBP - systolic blood pressure, AC - abdominal circumference, DM_NO- presence/absence of diabetes, MS_No - presence/absence of metabolic syndrome, TG - triglycerides, HDLHDL-cholesterol 
A. COZMA, A. FODOR, O. H. ORĂȘAN, R. SUHAROSCHI, C. MUREȘAN, R. VULTURAR,

D. SAMPELEAN, V. NEGREAN, D. POP, A. SITAR-TĂUT

Table 3. - Multivariate regression analysis for QUICKI index model summary and coefficients

\begin{tabular}{|c|c|c|c|c|c|c|c|}
\hline \multicolumn{5}{|c|}{ Model Summary } & & & \\
\hline Model & $\mathrm{R}$ & $\begin{array}{c}\mathrm{R} \\
\text { Square }\end{array}$ & $\begin{array}{l}\text { Adjusted R } \\
\text { Square }\end{array}$ & $\begin{array}{l}\text { Std. Error of } \\
\text { the Estimate }\end{array}$ & & & \\
\hline 1 & $.323^{\mathrm{a}}$ & .104 & .093 & .02740 & \multicolumn{3}{|c|}{ a. Predictors: (Constant), BMI } \\
\hline 2 & $.417^{\mathrm{b}}$ & .174 & .153 & .02648 & \multicolumn{3}{|c|}{ b. Predictors: (Constant), BMI, AC } \\
\hline 3 & $.536^{\mathrm{c}}$ & .288 & .261 & .02474 & \multicolumn{3}{|c|}{$\begin{array}{l}\text { c. Predictors: (Constant), BMI, AC, } \\
\text { DM_NO }\end{array}$} \\
\hline 4 & $.595^{\mathrm{d}}$ & .354 & .322 & .02370 & \multicolumn{3}{|c|}{$\begin{array}{l}\text { d. Predictors: (Constant), BMI, AC, } \\
\text { DM_NO, TG }\end{array}$} \\
\hline 5 & $.602^{\mathrm{e}}$ & .362 & .321 & .02371 & \multicolumn{3}{|c|}{$\begin{array}{l}\text { e. Predictors: (Constant), BMI, AC, } \\
\text { DM NO, TG, HDL }\end{array}$} \\
\hline 6 & $.609^{f}$ & .371 & .322 & .02370 & \multicolumn{3}{|c|}{$\begin{array}{l}\text { f. Predictors: (Constant), BMI, AC, } \\
\text { DM_NO, TG, HDL, MS_NO } \\
\text { g. Dependent Variable:QUICKI index }\end{array}$} \\
\hline \multicolumn{8}{|c|}{ Coefficients $^{a}$} \\
\hline \multirow{3}{*}{\multicolumn{3}{|c|}{ Model }} & \multicolumn{2}{|c|}{ Unstandardized Coefficients } & $\begin{array}{l}\text { Standardized } \\
\text { Coefficients }\end{array}$ & $\mathrm{t}$ & Sig. \\
\hline & & & B & Std. Error & Beta & & \\
\hline & & & .422 & .021 & & 20.388 & .000 \\
\hline 1 & \multicolumn{2}{|c|}{ (Constant) } & -.002 & .001 & -.323 & -3.087 & .003 \\
\hline \multirow[t]{3}{*}{2} & \multicolumn{2}{|c|}{ (Constant) } & .452 & .023 & & 19.685 & .000 \\
\hline & \multirow{2}{*}{\multicolumn{2}{|c|}{ BMI }} & .000 & .001 & .034 & .198 & .844 \\
\hline & & & -.001 & .000 & -.443 & -2.608 & .011 \\
\hline \multirow[t]{4}{*}{3} & \multirow{2}{*}{\multicolumn{2}{|c|}{$\begin{array}{l}\text { (Constant) } \\
\text { BMI }\end{array}$}} & .438 & .022 & & 20.104 & .000 \\
\hline & & & $-7.553 \mathrm{E}-005$ & .001 & -.011 & -.070 & .945 \\
\hline & & -.001 & .000 & -.323 & -1.990 & .050 \\
\hline & \multicolumn{2}{|c|}{ DM_NO } & -.028 & .008 & -.349 & -3.583 & .001 \\
\hline \multirow[t]{5}{*}{4} & \multicolumn{2}{|c|}{ (Constant) } & .442 & .021 & & 21.119 & .000 \\
\hline & \multirow{2}{*}{\multicolumn{2}{|c|}{$\begin{array}{l}\text { BMI } \\
\text { AC }\end{array}$}} & $-8.645 \mathrm{E}-005$ & .001 & -.013 & -.083 & .934 \\
\hline & & & -.001 & .000 & -.271 & -1.734 & .087 \\
\hline & \multicolumn{2}{|c|}{ DM_NO } & -.022 & .008 & -.274 & -2.819 & .006 \\
\hline & \multicolumn{2}{|c|}{$\mathrm{TG}^{-}$} & .000 & .000 & -.277 & -2.853 & .006 \\
\hline \multirow[t]{6}{*}{5} & \multicolumn{2}{|c|}{ (Constant) } & .421 & .030 & & 14.131 & .000 \\
\hline & BMI & & $-6.935 \mathrm{E}-005$ & .001 & -.010 & -.067 & .947 \\
\hline & AC & & -.001 & .000 & -.258 & -1.642 & .105 \\
\hline & DM_NC & & -.022 & .008 & -.282 & -2.892 & .005 \\
\hline & $\mathrm{TG}^{-}$ & & $-8.977 \mathrm{E}-005$ & .000 & -.243 & -2.361 & .021 \\
\hline & HDL & & .000 & .000 & .095 & .964 & .338 \\
\hline 6 & (Const & & .421 & .030 & & 14.142 & .000 \\
\hline & BMI & & $-5.263 \mathrm{E}-005$ & .001 & -.008 & -.051 & .960 \\
\hline & AC & & -.001 & .000 & -.230 & -1.440 & .154 \\
\hline & DM_NC & & -.021 & .008 & -.264 & -2.664 & .009 \\
\hline & $\mathrm{TG}^{-}$ & & $-7.924 \mathrm{E}-005$ & .000 & -.215 & -2.014 & .047 \\
\hline & $\mathrm{HDL}$ & & .000 & .000 & .068 & .668 & .506 \\
\hline & MS_NC & & -.007 & .007 & -.116 & -1.033 & .305 \\
\hline
\end{tabular}

BMI- body mass index, AC - abdominal circumference, DM_NO- presence/absence of diabetes, MS _ No- presence/absence of metabolic syndrome, TG - triglycerides, HDL- HDLcholesterol 
A COMPARISON BETWEEN INSULIN RESISTANCE

SCORES PARAMETERS IN IDENTIFYING PATIENTS WITH METABOLIC SYNDROME

Table 4. - Multivariate regression analysis for McAuley index model summary and coefficients

\begin{tabular}{|c|c|c|c|c|c|c|}
\hline \multicolumn{5}{|c|}{ Model Summary } & \multirow{2}{*}{\multicolumn{2}{|c|}{$\begin{array}{l}\text { a. Predictors: (Constant), } \\
\text { BMI }\end{array}$}} \\
\hline Model & $\mathrm{R}$ & $\begin{array}{c}\mathrm{R} \\
\text { Square }\end{array}$ & $\begin{array}{l}\text { Adjusted R } \\
\text { Square }\end{array}$ & $\begin{array}{l}\text { Std. Error of the } \\
\text { Estimate }\end{array}$ & & \\
\hline 1 & $.461^{a}$ & .212 & .203 & .40513 & \multicolumn{2}{|c|}{$\begin{array}{l}\text { b. Predictors: (Constant), } \\
\text { BMI, AC }\end{array}$} \\
\hline 2 & $.501^{\mathrm{b}}$ & .251 & .233 & .39743 & \multicolumn{2}{|c|}{$\begin{array}{l}\text { c. Predictors: (Constant), } \\
\text { BMI, AC, DM_NO }\end{array}$} \\
\hline 3 & $.572^{\mathrm{C}}$ & .327 & .302 & .37915 & \multicolumn{2}{|c|}{$\begin{array}{l}\text { d. Predictors: (Constant), } \\
\text { BMI, AC, DM_NO, HDL }\end{array}$} \\
\hline 4 & $.616^{\mathrm{d}}$ & .379 & .347 & .36649 & \multicolumn{2}{|c|}{$\begin{array}{l}\text { e. Predictors: (Constant), } \\
\text { BMI, AC, DM_NO, HDL, } \\
\text { MS_NO }\end{array}$} \\
\hline 5 & $.651^{\mathrm{e}}$ & .424 & .387 & .35515 & \multicolumn{2}{|c|}{$\begin{array}{l}\text { f Dependent Variable: } \\
\text { McAULEY score }\end{array}$} \\
\hline \multicolumn{7}{|c|}{ Coefficients $^{\mathrm{a}}$} \\
\hline \multirow{2}{*}{\multicolumn{2}{|c|}{ Model }} & \multicolumn{2}{|c|}{$\begin{array}{l}\text { Unstandardized } \\
\text { Coefficients }\end{array}$} & $\begin{array}{c}\text { Standardized } \\
\text { Coefficients }\end{array}$ & \multirow[t]{2}{*}{$t$} & \multirow[t]{2}{*}{ Sig. } \\
\hline & & $\mathrm{B}$ & Std. Error & Beta & & \\
\hline \multirow[t]{2}{*}{1} & (Constant) & 3.486 & .306 & & 11.381 & .000 \\
\hline & BMI & -.049 & .011 & -.461 & -4.699 & .000 \\
\hline \multirow[t]{3}{*}{2} & (Constant) & 3.831 & .344 & & 11.127 & .000 \\
\hline & BMI & -.021 & .017 & -.194 & -1.199 & .234 \\
\hline & AC & -.012 & .006 & -.332 & -2.052 & .043 \\
\hline \multirow[t]{4}{*}{3} & (Constant) & 3.653 & .334 & & 10.946 & .000 \\
\hline & BMI & -.025 & .017 & -.230 & -1.488 & .141 \\
\hline & AC & -.009 & .006 & -.234 & -1.483 & .142 \\
\hline & DM_NO & -.355 & .118 & -.284 & -3.000 & .004 \\
\hline \multirow[t]{5}{*}{4} & (Constant) & 2.876 & .442 & & 6.507 & .000 \\
\hline & BMI & -.024 & .016 & -.225 & -1.501 & .137 \\
\hline & AC & -.007 & .006 & -.186 & -1.210 & .230 \\
\hline & DM_NO & -.352 & .114 & -.282 & -3.080 & .003 \\
\hline & HDL̄ & .013 & .005 & .234 & 2.573 & .012 \\
\hline \multirow[t]{6}{*}{5} & (Constant) & 2.962 & .430 & & 6.893 & .000 \\
\hline & BMI & -.024 & .016 & -.220 & -1.518 & .133 \\
\hline & AC & -.004 & .006 & -.116 & -.763 & .448 \\
\hline & DM_NO & -.281 & .114 & -.225 & -2.456 & .016 \\
\hline & HDL & .008 & .005 & .155 & 1.650 & .103 \\
\hline & MS_NO & -.243 & .098 & -.256 & -2.475 & .015 \\
\hline
\end{tabular}

AC - abdominal circumference, DM_NO- presence/absence of diabetes, MS_ NO presence/absence of metabolic syndrome, TG - triglycerides, HDL- HDL-cholesterol

QUICKI was significantly negatively correlated with BMI (correlation coeff $=-0.323, p=0.003$ ), abdominal circumference (correlation coeff $=-0.416$, $p<0.001$ ), the presence of diabetes (correlation coeff $=-0.429, p<0.001$ ), glycemia (correlation coeff $=-0.530, p<0.001$ ), the TG value (correlation coeff $=-0.433$, 
A. COZMA, A. FODOR, O. H. ORĂȘAN, R. SUHAROSCHI, C. MUREȘAN, R. VULTURAR, D. SAMPELEAN, V. NEGREAN, D. POP, A. SITAR-TĂUT

$p=0.001$ ), the presence of MS (correlation coeff $=-0.417, p<0.001$ ), and was directly correlated with HDL-cholesterol (correlation coeff $=0.262, p=0.016$ ) For QUICKI, the independent predictors factors were found to be - diabetes (coefficient of determination $=-2.664, p=0.009$ ) and triglycerides (coefficient of determination $=-2.014, p=0.047$ ) (Table 3 ).

McAuley index was inversely correlated with $\mathrm{BMI}$ (correlation coeff $=$ $-0.461, p<0.001$ ), abdominal circumference (correlation coeff $=-0.488, p<0.001$ ), glycemia (correlation coeff $=-0.392, p<0.001$ ), the presence of DM (correlation coeff $=-0.375, p<0.001$ ), the serum TG value (correlation coeff $=-0.775$, $p<0.001$ ), the presence of MS (correlation coeff $=-0.507 p<0.001$ ), and was directly correlated with HDL (correlation coeff $=0.337, p=0.002$ ). For McAuley index, the independent predictors factors were found to be - diabetes (coefficient of determination $=-2.225, \mathrm{p}=0.016$ ) and metabolic syndrome (coefficient of determination $=-2.475, \mathrm{p}=0.015)($ Table 4$)$.

The determined areas under the ROC curve were as follows: 0.769 for HOMA, 0.769 for QUICKI index, 0.818 for McAuley Score. Diagnostic cut-off levels with optimum sensitivity and specificity were found to be 1.07 for HOMA (sensitivity $87.27 \%$, specificity $62.07 \%$ ), 2.14 for McAuley Score (sensitivity $81.82 \%$, specificity $75.86 \%$ ), 0.37 for QUICKI index (sensitivity $87.27 \%$, specificity $62.07 \%$ ) (Table 5 ).

Table 5. Areas under the ROC for HOMA, QUICKI, McAULEY indexes

\begin{tabular}{|c|c|c|c|c|c|c|c|}
\hline $\begin{array}{l}\text { AUROC } \\
(95 \% \mathrm{Cl})\end{array}$ & & HOMA & QUICKI & McAuley & $P^{*}$ & $P^{* *}$ & $P^{* * *}$ \\
\hline & Global & $\begin{array}{l}0.769 \\
(0.664-0.854)\end{array}$ & $\begin{array}{l}0.769 \\
(0.664-.854)\end{array}$ & $\begin{array}{l}0.818 \\
(0.718-0.893)\end{array}$ & NS & NS & NS \\
\hline & Women & $\begin{array}{l}0.736 \\
(0.610-0.839)\end{array}$ & $\begin{array}{l}0.736 \\
(0.609-0.839)\end{array}$ & $\begin{array}{l}0.837 \\
(0.723-0.918)\end{array}$ & NS & 0.034 & 0.04 \\
\hline & Men & $\begin{array}{l}0.947 \\
(0.754-0.992)\end{array}$ & $\begin{array}{l}0.947 \\
(0.754-0.992)\end{array}$ & $\begin{array}{l}0.737 \\
(0.502-0.901)\end{array}$ & NS & NS & NS \\
\hline
\end{tabular}

$P^{*}$ - between HOMA and QUICKI; $P^{* *}$ - between HOMA and McAuley; $P^{* \star *}$ - between QUICKI and McAuley

Subsequently, we calculated the cut-off values for the 3 IR indexes: HOMA, QUICKI and McAuley, globally (Figure 1), in women (Figure 2). Overall, the highest specificity was found for McAuley index (75.86\%). By analyzing separately in women the cut-off values of the IR indexes, their 
sensitivity and specificity, respectively, it was found that in women, McAuley index had the highest sensitivity (97.2\%). It was not possible to performe a detailed analyses for men, because the number of males without MS was very small.
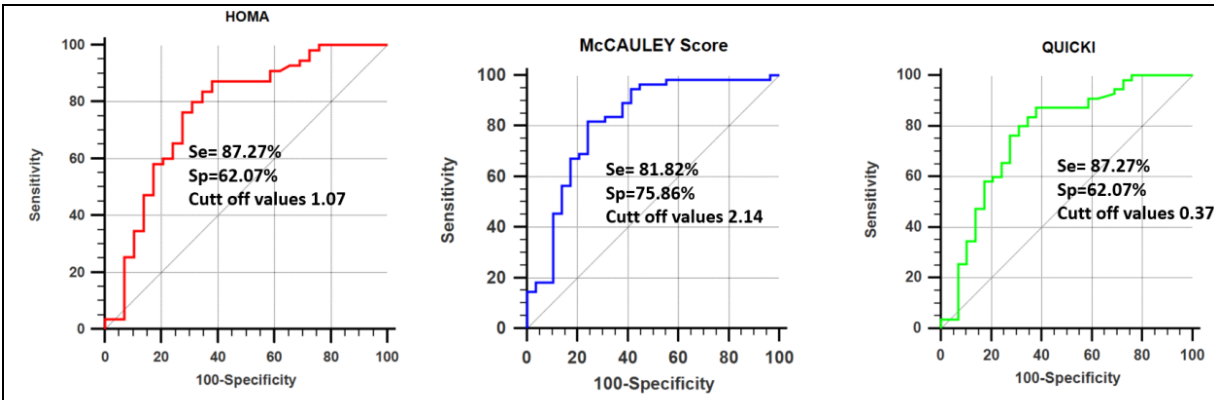

Figure 1. - AUROCs, Se, Sp and cut-off values - globally
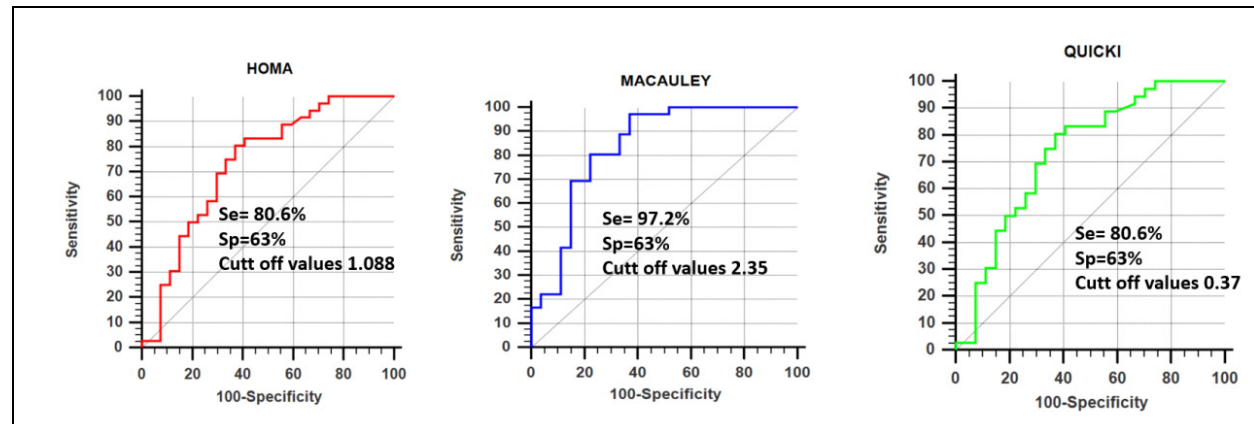

Figure 2. - AUROCs, Se, Sp and cut-off values in women

Although other authors [15] consider that the insulin resistance diagnosis is established at a HOMA index value $>2.7$ [16], in our study, the cut-off value for the diagnosis of MS was 1.07.

Cut-off values differ depending on the definition of MS; thus, GayosoDiz et al. [14] showed that using the IDF definition, like in our study, the ROC value was 0.69 , and using the ATPIII definition, the ROC value was 0.72 .

These results are similar to the study conducted by Esteghamati, who found an AUC of $0.65(0.63,0.67)$ for IDF and $0.68(0.66,0.70)$ for ATPIII [17]. In the current study, the ROC value was 1.07 for the HOMA index. 
A. COZMA, A. FODOR, O. H. ORĂȘAN, R. SUHAROSCHI, C. MUREȘAN, R. VULTURAR,

D. SAMPELEAN, V. NEGREAN, D. POP, A. SITAR-TĂUT

HOMA-IR is a reliable surrogate method to estimate $I R$ in an epidemiological or clinical setting. However, its threshold levels vary widely; the cut-off values of HOMA-IR are usually defined by population-based percentile criteria. Moreover, these cut-off values are different depending on ethnicity, clinical methods of estimation, and metabolic conditions of the studied populations $[18,19]$.

In the Spanish population, the threshold value of HOMA-IR decreases from 3.46 using 90th percentile criteria [20] to 2.05 taking into account the MetS components. Our HOMA-IR cut-off levels are relatively low compared to those reported in a study on healthy Italian patients [21], with a value of 2.77, and in a Spanish non-diabetic population [22], with a value of 3.8.

Gayoso-Diz et al. [14] concluded that the effect of age and gender on the ability of HOMA-IR to identify subjects with a cardiometabolic risk phenotype should be taken into account when estimating its values in different populations.

Shalaurova [23] showed in a study that the IR score was strongly correlated with triglycerides $(r=0.74)$ and fasting insulin levels $(r=0.51)$, and was inversely correlated with HDL-C ( $r=-0.67)$, like in the current study, where the best correlation with TG was found for McAuley index.

Tosi et al. [24] reported that the insulin resistance indexes HOMA and QUICKI had a sensitivity of 50.9 and 57.7 , respectively, in identifying insulin resistance, significantly lower compared to the current study, while their specificity was 88.3 , and 86.2 , respectively, AUC for HOMA and QUICKI being 0.798 . In our study, the specificity of these indexes in identifying patients with MS was lower, and AUC was higher. The authors of this study concluded that these IR indexes were very well correlated with the hyperinsulinemiceuglycemic clamp, which is the gold standard in IR diagnosis. McAuley index has the highest sensitivity, in women. An important element in the current study is the fact that independent predictors for the insulin resistance indexes are different: for HOMA: diabetes, for QUICKI: diabetes and triglycerides and for McAuley: diabetes and metabolic syndrome.

\section{CONCLUSIONS}

The measurement of IR remains a challenge. The current gold standard test is the hyperinsulinemic-euglycemic clamp technique, in which a constant rate of insulin infusion is balanced with concomitant variable glucose infusion to maintain euglycemia; it is an invasive time- and labor-intensive approach - not suitable for epidemiological and diagnostic studies. Although HOMA is more commonly used in practice (probably due to its easy use), McAuley Score seems to have a better specificity in identifying patients with MetS. In women, McAuley index has the highest sensitivity. 


\section{EXPERIMENTAL SECTION}

The study was carried out at the $4^{\text {th }}$ Department of Internal Medicine, Cluj-Napoca; The rights of the patients regarding the confidentiality of personal information were respected in agreement to Helsinki declaration of Ethical Principles for Medical Research Involving Human Subjects.

84 consecutive participants completed a questionnaire regarding their personal and family medical history. All were subjected to a complete physical exam.

For each patient, weight, height and abdominal circumference (midway between the inferior margin of the last rib and the iliac crest in horizontal plane while in upright position) were measured. The body mass index (BMI) was calculated (weight $(\mathrm{kg}) /[$ height $(\mathrm{m})] 2$ ); subjects with a $\mathrm{BMI} \geq 30 \mathrm{~kg} / \mathrm{m} 2$ were considered obese.

Blood samples were collected after an overnight fast of $>8 \mathrm{~h}$. Plasma glucose levels were measured using a hexokinase enzymatic reference method. Fasting insulin levels were measured using a radioimmunoassay method or ELISA. Fasting lipids were analyzed, and for the present study, serum levels of cholesterol $\geq 5.172 \mathrm{mmol} / \mathrm{L}$ and triglycerides $\geq 1.7 \mathrm{mmol} / \mathrm{L}$ were considered abnormal.

The diagnosis of MetS was made based on International Diabetes Federation criteria, including the presence of abdominal obesity (waist circumference $\geq 94 \mathrm{~cm}$ for men and $\geq 80 \mathrm{~cm}$ for women) plus at least two of the following criteria: 1) fasting glucose $\geq 100 \mathrm{mg} / \mathrm{dL}$; 2) systolic blood pressure $\geq 130 \mathrm{mmHg}$ or diastolic blood pressure $\geq 85 \mathrm{mmHg}$ or treatment of previously diagnosed hypertension; 3) HDL-cholesterol $<40 \mathrm{mg} / \mathrm{dL}$ for men and $<50 \mathrm{mg} / \mathrm{dL}$ for women or treatment with high-dose omega-3 therapy; 4) triglycerides $\geq 150 \mathrm{mg} / \mathrm{dL}$ or drug treatment with fenofibrate.

Insulin resistance was assessed by:

$$
\begin{aligned}
& \text { - } \quad \text { HOMA-IR = insulin }(\mu \mathrm{U} / \mathrm{mL}){ }^{*} \text { glycemia }(\mathrm{mg} / \mathrm{dl}) / 405 ; \\
& \text { - } \quad \text { MCACKI =1 / [log (insulin }(\mu \mathrm{U} / \mathrm{mL}))+\log (\text { glycemia) }] ; \\
& 0.28 \text { * log (triglyceride)]; }
\end{aligned}
$$

The blood insulin level can be expresed in international units, such as $\mu \mathrm{IU} / \mathrm{mL}$ or in molar concentration, a typical blood level between meals is 8-11 $\mu \mathrm{lU} / \mathrm{mL}$ (57-79 pmol/L). Statistical analysis - the data were analyzed using SPSS 16.0 for Windows and MedCalc 10.3.0.0 software programs. Descriptive statistics was performed. Data's normality was assessed using Kolmogorov-Smirnov test. Numerical data were compared using Student or Mann-Whitney test; for categorical variable $\mathrm{x} 2$ test was used. Univariate and 
A. COZMA, A. FODOR, O. H. ORĂȘAN, R. SUHAROSCHI, C. MUREȘAN, R. VULTURAR,

D. SAMPELEAN, V. NEGREAN, D. POP, A. SITAR-TĂUT

multivariate analysis was performed. Cut-off values, optimum sensitivity, specificity and area under the receiver operating characteristic (ROC) curve were evaluated. $p<0.05$ was considered significantly statistic.

\section{ACKNOWLEDGMENTS} 2246.

Authors greatly acknowledge the financial support offered by GRANT ID

\section{REFERENCES}

1. Bertoni A.G., Wong N.D., Shea S., Ma S., Liu K, Preethi S., Jacobs D.R., Wu C., Saad M.F., Szkio M., Diabetes Care, 2007, 30, 2951-2956.

2. Montecucco F., Steffens S., Mach F., Mediators of Inflammation, 2008, 767623.

3. Tenenbaum A., Adler Y., Boyko V., American Heart Journal, 2007; 153, 559.

4. Verhagen S.N., Wassink A.M., van der Graaf Y., Gorter P.M., Visseren F.L., Cardiovascular Diabetology, 2011; 10, 100.

5. Zuliani G., Morieri M.L., Volpato S, Atherosclerosis, 2014; 235, 538.

6. Nasr G., Sliem H., The International Journal of Cardiovascular Imaging 2011; $27,335$.

7. Gazzaruso C., Solerte S.B., De Amici E., The American Journal of Cardiology, 2006; 97: 236-239.

8. Tataru D.A., Olinic D.M., Urda A., Olinic M., Orasan R., Studia UBB Chemia, 2018, 62, 127.

9. Alberti, K.G., P. Zimmet and J. Shaw, Diabetic Medicine, 2006, 23(5) 469.

10. Matthews, D.R., J.P. Hosker, A.S. Rudenski, B.A. Naylor, D.F. Treacher and R.C. Turner, Diabetologia, 1985, 28(7) 412.

11. Katz, A., S.S. Nambi, K. Mather, A.D. Baron, D.A. Follmann, G. Sullivan and M. J. Quon, The Journal of Clinical Endocrinology and Metabolism, 2000, 85(7) 2402.

12. McAuley K.A., Williams S.M., Mann J.I., Walker R.J., Lewis-Barned N.J., Temple L.A., Duncan A.W., Diabetes Care, 2001, 24(3), 460.

13. Abbasi, F., D. Shiffman, C.H. Tong, J.J. Devlin and M.J. McPhaul, Journal of the Endocrine Society, 2018, 2(9),1050.

14. Gayoso-Diz, P., A. Otero-Gonzalez, M.X. Rodriguez-Alvarez, F. Gude, F. Garcia, A. De Francisco and A.G. Quintel, BMC Endocrine Disorders, 2013, $13,47$.

15. Carvalho C., Nutrition, 2018, https,//doi.org/10.1016/j.nut.2018.07.014.

16. Geloneze B., Vasques A.C., Stabe C.F., Pareja J.C., Rosado L.E., Queiroz E.C., Arquivos Brasileiros de Endocrinologia \& Metabologia, 2009, 53, 281-7. 
17. Esteghamati A., Ashraf H., Khalilzadeh O., Zandieh A., Nakhjavani M., Rashidi A., Haghazali M., Asgari F., Nutrition \& Metabolism, 2010, 7, 26.

18. Antuna-Puente B., Disse E., Rabasa-Lhoret R., Laville M., Capeau J., Bastard, Diabetes \& Metabolism, 2011, 37, 179.

19. Saplontai-Pop A., Moldovan M., Oprean R. Orasan. O., Saplontai S., Ionescu C., Studia UBB Chemia, 2014, 59(4), 39.

20. Gayoso-Diz P., Otero-González A., Rodriguez-Alvarez M.X., Gude F., Cadarso-Suarez C., García F., De Francisco A, Diabetes Research and Clinical Practice, 2011, 94, 146.

21. Miccoli R., Biamchi C., Odoguardi, Nutrition, Metabolism and Cardiovascular Diseases, NMCD, 2005, 15, 250.

22. Ascaso J.F., Romero P., Real J.T., Priego A., Valdecabres C., Carmena R. Medicina Clinica (Barc), 2001, 117, 530.

23. Shalaurova I., Connelly M.A., Garvey T.W., and Otvos D.J., Metabolic syndrome and related disorders, 2014, (12) 8, 422.

24. Tosi F., Bonora E., Paolo Moghetti P, Human Reproduction, 2017, 32(12), 2515. 
